\title{
DECISION SUPPORT AND GEOGRAPHICAL INFORMATION SYSTEMS
}

\author{
Kurt Fedra \\ International Institute for Applied Systems Analysis \\ Laxenburg, Austria \\ René $\mathrm{F}$. Reitsma \\ Katholieke Universiteit Nijmegen \\ Nijmegen, The Netherlands \\ RR-90-9 \\ December 1990
}

Reprinted from H.J. Scholten and J.C.H. Stillwell (eds.), Geographical Information Systems for Urban and Regional Planning, 177-188.

INTERNATIONAL INSTITUTE FOR APPLIED SYSTEMS ANALYSIS Laxenburg, Austria 
Research Reports, which record research conducted at IIASA, are independently reviewed before publication. However, the views and opinions they express are not necessarily those of the Institute or the National Member Organizations that support it.

Reprinted from H.J. Scholten and J.C.H. Stillwell (eds.), Geographical Information Systems for Urban and Regional Planning, 177-188.

Copyright (C) 1990 Kluwer Academic Publishers

All rights reserved. No part of this publication may be reproduced or transmitted in any form or by any means, electronic or mechanical, including photocopy, recording, or any information storage or retrieval system, without permission in writing from the copyright holder.

Printed by Novographic, Vienna, Austria 


\section{Foreword}

Central to IIASA's function as an institute of applied systems analysis is the requirement to provide the tools, information, and advice needed by those who face and must respond to real world issues, problems, and opportunities.

Many of these issues, problems, and opportunities have a spatial dimension, and it is the interface and integration of decision support tools and geographical information systems that this report addresses. Spatially distributed information, problems, and solutions, require special tools of analysis.

The research described in this report results from a series of research and development projects, carried out by IIASA's Advanced Computer Applications group.

The examples presented range from work done for the Commission of the European Communities' Joint Research Centre and the Dutch Ministry for Housing, Physical Planning and the Environment in the area of technological and environmental risk analysis; the US Bureau of Reclamation in a project on groundwater management; the French Transportation research Institute INRETS, in a project on hazardous goods transportation risk analysis; and the State Science and Technology Commission of the People's Republic of China in a project on regional development planning.

Using computer graphics for the visualization of information, problems, and solutions and as the basis for easy-to-use software tools, the research results discussed in this report demonstrate the role and potential of customized results of general systems analysis and modeling - which, by its usefulness and relevance, emphasizes IIASA's growing utility to a widening constituency. 


\title{
16 DECISION SUPPORT AND GEOGRAPHICAL INFORMATION SYSTEMS
}

\author{
Kurt Fedra and Rene F. Reitsma
}

\subsection{Introduction}

Geographical Information Systems (GIS) are gaining increasing importance and widespread acceptance as tools for decision support in land, infrastructure, resources, environmental management and spatial analysis, and in urban and regional development planning. GIS assist in the preparation, analysis, display, and management of geographical data. It is in the analysis and display functions that GIS meet Decision Support Systems (DSS). DSS analyse and support decisions through the formal analysis of alternative options, their attributes vis-a-vis evaluation criteria, goals or objectives, and constraints. DSS functions range from information retrieval and display, filtering and pattern recognition, extrapolation, inference and logical comparison, to complex modelling. The use of model-based information and DSS, and in particular of interactive simulation and optimization models that combine traditional modelling approaches with new expert systems techniques of Artificial Intelligence (AI), dynamic computer graphics and geographical information systems, is demonstrated in this chapter with application examples from technological risk assessment, environmental impact analysis, and regional development planning. With the emphasis on an easy-to-understand visual problem representation, using largely symbolic interaction and dynamic images that support understanding and insight, these systems are designed to provide a rich and directly accessible information basis for decision support and planning.

\subsection{Decision support}

Underlying the concept of DSS in general is the recognition that there is a class of decision problems that is neither well structured nor unambiguous. Such problems cannot be properly solved by a single systems analysis effort or a highly structured computerized decision aid (Fick and Sprague 1980). They are not unique so a one-shot effort would be justified given that the problem is big enough. Neither do they recur frequently enough with sufficient similarity to subject them to rigid mathematical treatment. They are somewhere in between. Due to the mixture of uncertainty in the scientific aspects of the problem, and the subjective and judgmental elements in its socio-political aspects, there is no wholly objective way to find a best solution.

There is no universally accepted definition of DSS. Almost any computer-based system, from database management or information systems via simulation models to mathematical programming or optimization, could conceivably support decisions. The literature on information systems and DSS is overwhelming; approaches range from a rigidly mathematical treatment to applied computer sciences, management sciences, or psychology. Decision support paradigms include predictive models, which give unique answers but with limited accuracy or validity. Scenario analysis relaxes the initial assumptions by making them more conditional, but at the same time more dubious. Normative models prescribe how things should happen, based on some theory, and generally involve optimization or game theory. Alternatively, descriptive or behavioural models supposedly describe things as they are, often with the exploitation of statistical techniques. Most recent assessments of the field, and in particular those concentrating 
on more complex, ill-defined, policy-oriented and strategic problem areas, tend to agree on the importance of interactiveness and the direct involvement of the end user. Direct involvement of the user results in new layers of feedback structures (Figure 16.1). The information system model is based on a sequential structure of analysis and decision support i.e. the relationships shown in the upper part of Figure 16.1. In comparison, the decision support model implies feedbacks from the applications, e.g. communication, negotiation, and bargaining to the information system, scenario generation, and strategic analysis. The realism of formal models is increased, for example, by the introduction of multiattribute utility theory (Keeney and Raiffa 1976, Bell et al. 1977), extensions including uncertainty and stochastic dominance concepts (e.g. Sage and White 1984), by multiobjective, multicriteria optimization methods, and finally by replacing strict optimization, requiring a complete formulation of the problem at the outset, by the concept of satisficing (Wierzbicki 1983).

Figure 16.1: Strategic decision problems: information systems versus DSS approach (partly after Radford 1978)
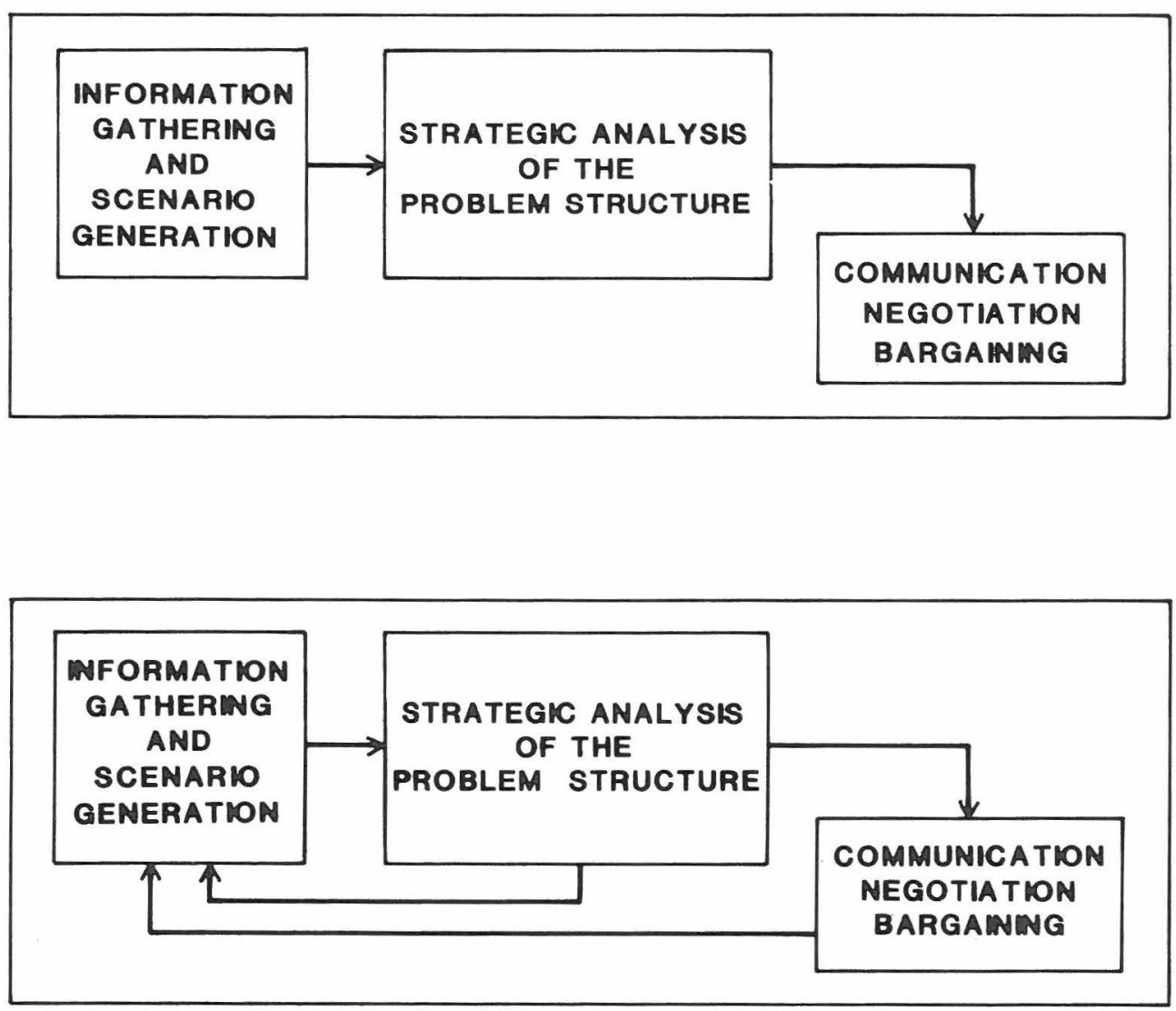
Another basic development is that of getting closer to the users. Interactive models and computer graphics are obvious developments here (e.g. Fedra and Loucks 1985). Decision conferences (Phillips 1984) are another approach, useful mainly in the early stages for the clarification of an issue. While certainly interactive in nature, most methods involve a decision analyst as well as a number of specialists (generally supposed to be the problem holders). Concentrating on the formulation of the decision problem, design and evaluation of alternatives, i.e. the substantive models, are only of marginal importance. Often enough, however, the problem holder (e.g. a regulatory agency) is not specialized in all the component domains of the problem (e.g. regional planning, environmental sciences, etc.). Expertise in the numerous domains touched upon by the problem situation is therefore as much a bottleneck as the structure of the decision problem. Building human expertise and some degree of intelligent judgement into decision supporting software is one of the major objectives of AI. Only recently has the area of expert systems or knowledge engineering emerged as a medium for successful and useful applications of AI techniques (see for example, Pearl et al. 1982, Sage and White 1984, or O'Brian 1985, Zimmermann 1987, Doukidis et al. 1989, on expert systems for decision support). An expert system is a computer program that is supposed to help solve complex real-world problems, in particular, specialized domains (e.g. Barr and Feigenbaum 1982). These systems use large bodies of domain knowledge, i.e. facts, procedures, rules and models, that human experts have collected or developed and found useful to solve problems in their domains.

\subsection{Geographical information systems}

Geographical information systems are tools to collect, store, retrieve, transform, analyse, and display spatial data. In many applications, it is the automated mapping and cartography, and the basic collection, organization, and management of spatial data that are of primary importance. However, with GIS' capability for analysis and display, and, in a limited sense, modelling, they can be regarded as a special class of decision support systems. Nevertheless, it is primarily in combination with simulation and optimization models, data bases of non-spatial data, AI, and in particular, expert systems technology, and decision support tools proper, that GIS become an extremely powerful and important building block of modern information technology.

\subsection{Application examples: integrated systems}

As discussed above, it is the integration of GIS and DSS, in combination with simulation or optimization models, related databases, and expert systems tools, that makes attractive and user-friendly decision support tools for a large spectrum of planning and management problems. Several examples of such systems that have been developed at IIASA over the last few years for application areas such as industrial risk assessment, hazardous substances management, environmental impact analysis and regional development planning are introduced below.

\section{Information Retrieval and Display}

Simulation and optimization models and model-based decision support tools tend to have considerable data requirements, which, in any particular application, may be a substantial problem for the effective use of these tools. They are therefore coupled, wherever feasible, to a number of data and information bases that provide not only the necessary background information to the user, but allow for the direct and automatic downloading of the 
model-relevant data. Obviously, for any problem with a spatial dimension, GIS count among the important information sources. For many problems, simple visualization, i.e. the graphical interpretation and display of the problem's structure and components, is an important aid to the decision maker. Translating complex and often abstract concepts such as risk into pictorial representation, and displaying spatial, temporal, and causal relationships in graphical formats often allows a more intuitive understanding of a problem. Pattern, or 'Gestalt', supporting understanding, emerges from graphical interpretation where narrative or numerical formats simply cannot convey the essential features of a problem situation.

A number of databases have been implemented and integrated with interactive browsing and display programs, problem-specific retrieval mechanisms, graphical display options, or linkage to several simulation models in the systems discussed below. While they use and process output, and in particular maps from standard GIS combined with other databases, they offer only a small but very problem-specific subset of GIS functionality in a restrictive but highly efficient menu-driven environment.

As part of a Europe-wide risk management system (Fedra 1985, Fedra and Otway 1986, Fedra et al. 1987) digital maps were used as a background for data display at various levels of aggregation ranging from the European level to very detailed country maps of, for example, the Netherlands. The contents of various databases used in one or several of the simulation models as well as spatial modelling results, can be viewed as interactively constructed map overlays. They include, for example, at the European level, political boundaries, major settlements, highway and national road networks, major industrial plant locations, chemical storage facilities and major water bodies.

In a risk analysis project for the Dutch government (see later section) where a very detailed set of maps with a basic resolution of 1:25,000 was used, features in addition to land use classes and surface waters include the transportation network with rail, road, and canals, city boundaries, or pipelines and power lines, population density, or weather data (windspeed and direction distributions) from individual weather stations. In total, about 50 attribute codes for different area and line features were used. For the raw digital data of approximately $40 \mathrm{MB}$ (vector maps in Digital Line Graph (DLG) format), a number of map manipulation and display routines were developed that allow the efficient generation of various overlay topics as well as arbitrary zooming into the maps (Plate 16.1).

Simulation models can also use digital maps or satellite images as backgrounds for the display of modelling results, or as a guiding framework for spatial problem descriptions. The maps, however, are only pictorial background and are not exploited as data sources. An example is the interactive groundwater modelling system, based on a 2D finite-element simulator (Fedra and Diersch 1989). Combining a finite-element model for flow and transport problems with an AI-based and symbolic, graphics user interface, the system is designed to allow the easy and efficient use of complex groundwater modelling technology in a problem- rather than model-oriented style. Implemented on a colour-graphics engineering workstation, the system provides a problem manager that allows the selection of site-specific, as well as generic, groundwater problems from problem libraries, or the interactive design of a new problem using a map as a background for the definition of problem geometry and hydrogeological parameters (Plate 16.2). With either satellite imagery (such as LANDSAT or SPOT) or DLG standard vector maps as a background, these problems can be edited and modified and then simulated under interactive user control. 


\section{Modelling and decision support for spatial problems}

A more complex combination of GIS outputs and features is in the functional coupling of spatial data and spatial simulation and optimization models as part of integrated decision support systems. Here data from the GIS are not only displayed, but used in the model-based analysis, with spatial results again displayed and possibly further analysed and interpreted with GIS components.

\section{Regional industrial structure optimization}

Industrial structure can be described as an interconnected and possibly spatially distributed network of complementary and alternative production technologies, their technological and economic properties, resource consumption, investment requirements, and the environmental consequences of production, and of course, location. DSS for industrial development planning (e.g. Fedra, Karhu et al. 1987, Fedra, Li et al. 1987, Zebrowski et al. 1988) are based on an optimization model that describes the behaviour of a given group of industries, under certain assumptions about prices for products, raw materials and labour, and upper and lower limits for certain production capacities or waste products. The industry will maximize its net economic results while meeting the external constraints, by adjusting production technologies and production capacities, resulting in a different product mix with different effects on the environment.

This approach can now be extended to include spatial aspects: different locations in a region or country have different sensitivities to environmental pollution, and to risk. As an example, consider the population density around a production plant, its location in relation to important water bodies used for various water supply purposes, etc. In addition, there are of course other important spatial considerations such as transportation costs, risks, and capacity constraints between the individual locations and the sources or markets for raw materials and products. The availability of the necessary technical infrastructure is also a spatial characteristic. Implemented as part of a case study of regional industrial development in China (Fedra, Li et al. 1987), the model considers a set of major production sites and external markets, and more than 140 alternative technologies. The model simultaneously considers criteria such as net and gross production value, export of key commodities (coal and electricity), production cost, domestic and foreign investment, resource consumption, and wastes generated. Local (i.e. spatially distributed) constraints include the availability of certain technologies at a given site, capacity constraints, resources such as coal, water and electricity, and the available labour force. For a given scenario in terms of technologies available, desired product mix and production levels, targets or constraints on the global objectives, and the local site-specific constraints, the model will produce the optimal selection, spatial allocation, and capacity of technologies, if a feasible solution exists at all.

\section{Technological risk analysis}

In a study for the Dutch Ministry for Housing, Physical Planning and the Environment (VROM), the Advanced Computer Applications (ACA) project developed an interactive and graphics-oriented framework and post-processor for the risk assessment package SAFETI (Technica 1984) to facilitate the quick generation, display, evaluation and comparison of policy alternatives and individual scenarios. The SAFETI package is a computer-based system for risk analysis of process plants. Based on the detailed geographical background data, the graphical interface to SAFETI's databases and consequence modelling results allows the display of the raw data such as plant locations, weather data, population distribution as thematic overlays over 
a basic land use map. Most of these data are also used for the computations of the risk estimates for individual plants or transportation problems along any of the transportation networks of the system (rail, road, canals). Once risk analysis has been performed for a specific process plant, the results are available for graphical display and interpretation. Risk contours can be displayed as transparent overlays on a map of the Netherlands. This map allows arbitrary zooming to provide the appropriate level of detail and resolution for a given decision problem.

\section{Air quality modelling}

For the regional to local scale, and for continuous emissions, a Gaussian air quality model for multiple point and area sources was adapted. The implementation example described below was designed and implemented for industrial centres in the People's Republic of China. The model was designed as a post-processor for production or energy scenarios based on coal and the regional industrial structure optimization model described above, assessing environmental impacts at a set of locations (areas up to 50 by 50 kilometres), characterized by a number of industrial point and area sources as well as domestic area sources. It translates emission characteristics for these sources into ambient $\left(\mathrm{SO}_{2}\right)$ concentrations for a user-defined weather situation. The model provides information on the feasibility and desirability of a given development scenario in terms of selected environmental impacts as one component of the regional development DSS.

From the GIS and related databases, the model constructs a site-specific data file, that is used for display as well as for the calculations, characterizing for one location (industrial installation or zone) the location of the individual sources as well as the default values of emission characteristics. Where available, a background land use map or satellite data are used. On the same grid, elevation data (e.g. from a digital elevation model) used for the dispersion model is stored. The model interface lists the point and area sources and displays a background map of the area studied with the location of the sources indicated. Model results are shown as a colour-coded pollutant concentration overlay on this map, a histogram (using the same colour code) of the frequency distribution of concentration values, or as a 3D concentration field displayed over the tilted and rotated image as illustrated in Plate 16.3.

\section{Transportation risk analysis}

In collaboration with INRETS-DERA, France, an information and decision support system for transportation risk assessment of dangerous goods was developed. The system improves availability and fast access to relevant data and information in adequate form (e.g. as topical maps), to support decisions on route selection or modes of transportation. Two kinds of raw data were available for inclusion in the system:

(i) spatial data: which could be represented as a map, in a vector or raster format, by data on land use, administrative boundaries (communes), road and railway network, and hydrological data; and

(ii) non-spatial data: mostly statistical, which provided the values of attributes of spatial entities (data on the transportation network and its surroundings, commune inventory, and the geographical index for agriculture, as well as data on products and firms concerned with production, usage or shipping of dangerous goods).

The aim was to create a general data structure able to handle these two kinds of data, which could be issued from different sources under different formats, and to connect a geographical 


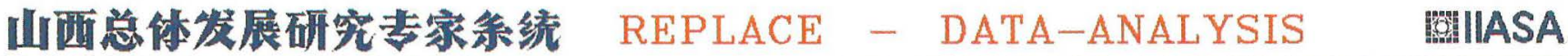

Attribute: population

Frequencies in 50 equal intervals

Min. value:

48547.00

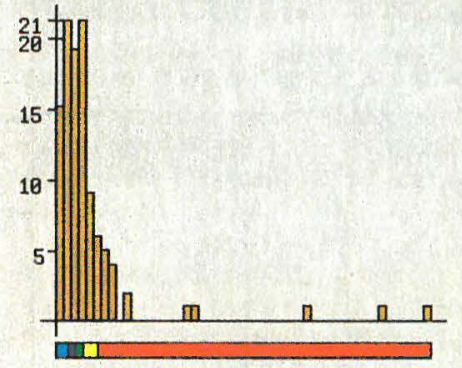

Max. value:

2713984.00

Sum:

30915028.00

Mean value: 288925.50

Median value: 206380.00

St.dev. $\quad 380072.16$

St.dev./mean: 1.32

Missing cases: 0

Frequencies in current classification Cumulative frequencies
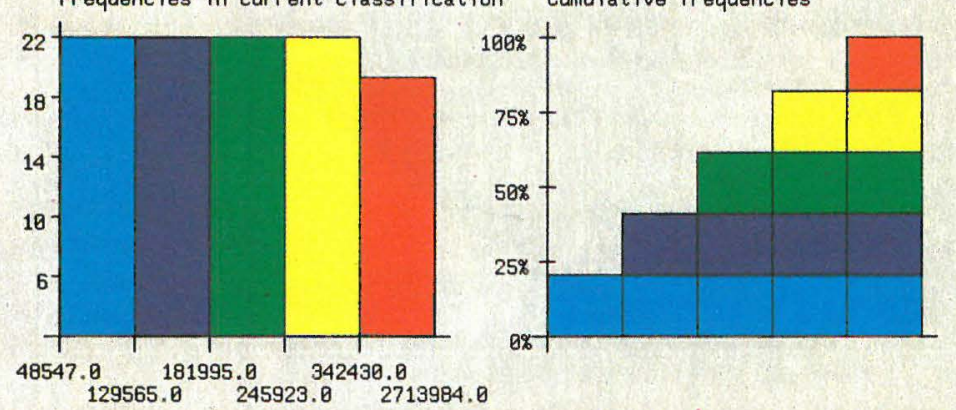

$129565.8 \quad 245923.8272430 .8$

\begin{tabular}{l} 
DAIA DISPLAY AND ANALYSIS MENU \\
Select and display attribute \\
Reclassify the attribute \\
\hline Compute Bi-Variate statistics \\
\hline EXPLAIN CURRENT OPTIONS \\
\hline RETURN TO UPPER LEVEL \\
\hline
\end{tabular}

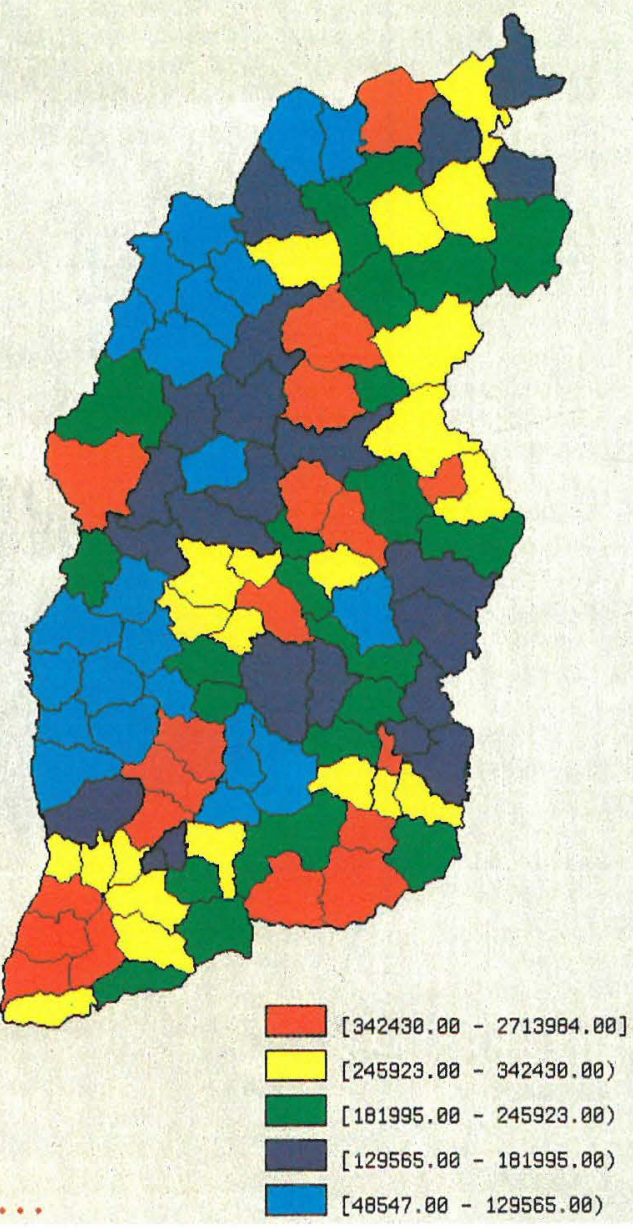




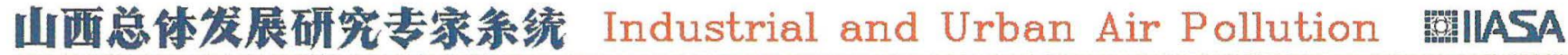

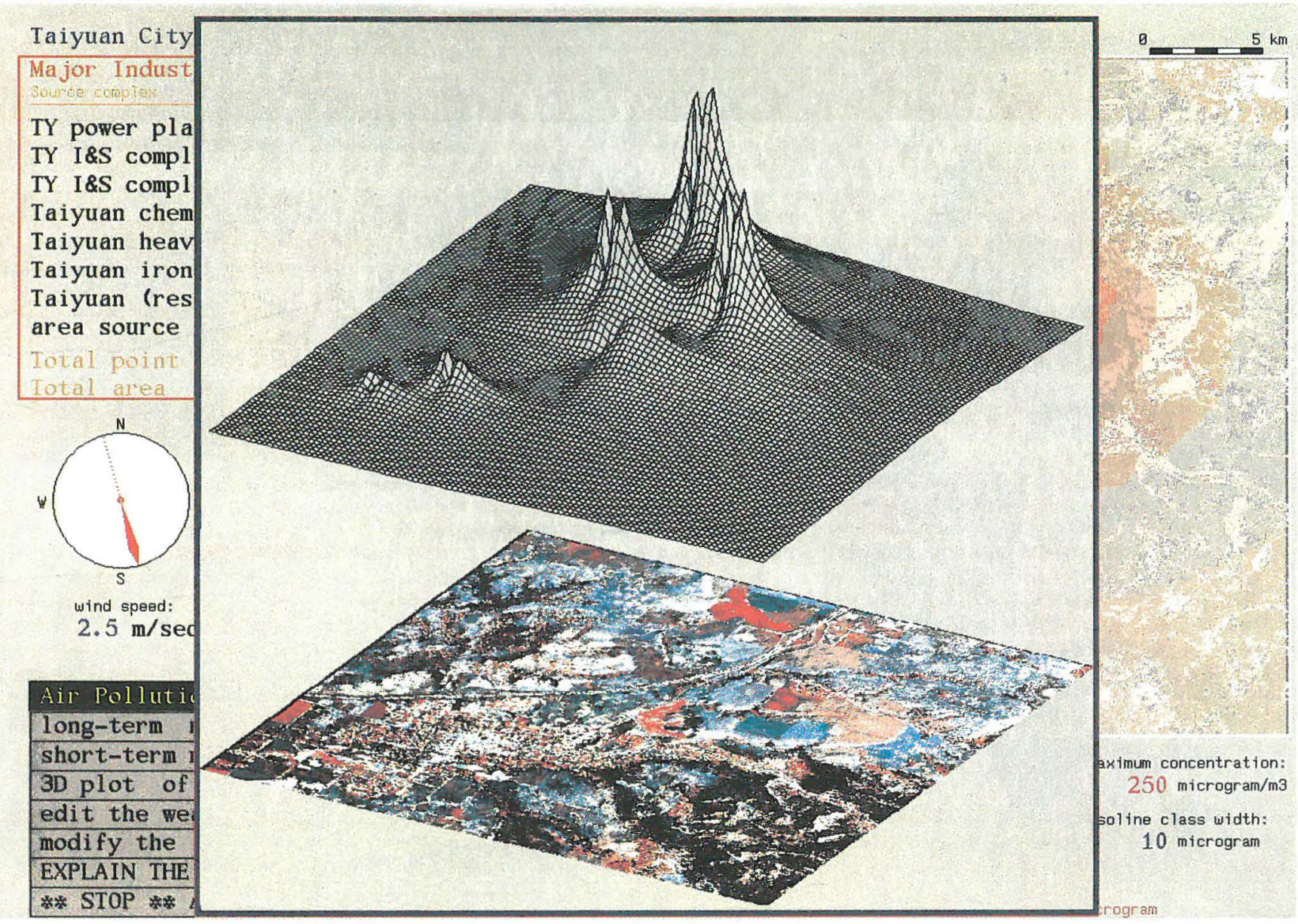

press any mouse button to continue... 


\section{FEFLOW FINITE ELEMENT MESH EDITOR AND GENERATOR IOIIASA}

PROBLEM TITLE: New FEFLOW-CAD problem DIMENSION: $8000 \mathrm{~m}$

BACKGROUND MAP: River_city_color Current Super Element 4 Nodes set: 3 Nodes to set: 1 X-Coor: $\quad 2545.650 \mathrm{~m}$ Y-Coor: $2005.750 \mathrm{~m}$

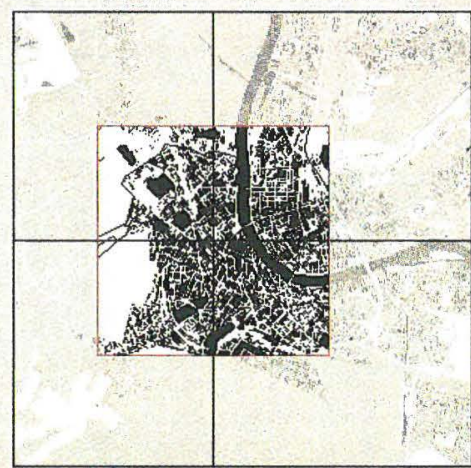
\begin{tabular}{l}
\hline FEFLOW MESH EDITOR MENL: \\
\hline Continue the mesh design \\
\hline Correct superelement mesh \\
\hline Zoom into background map \\
\hline Start the mesh generator \\
\hline RETURN / restore full map \\
\hline
\end{tabular}

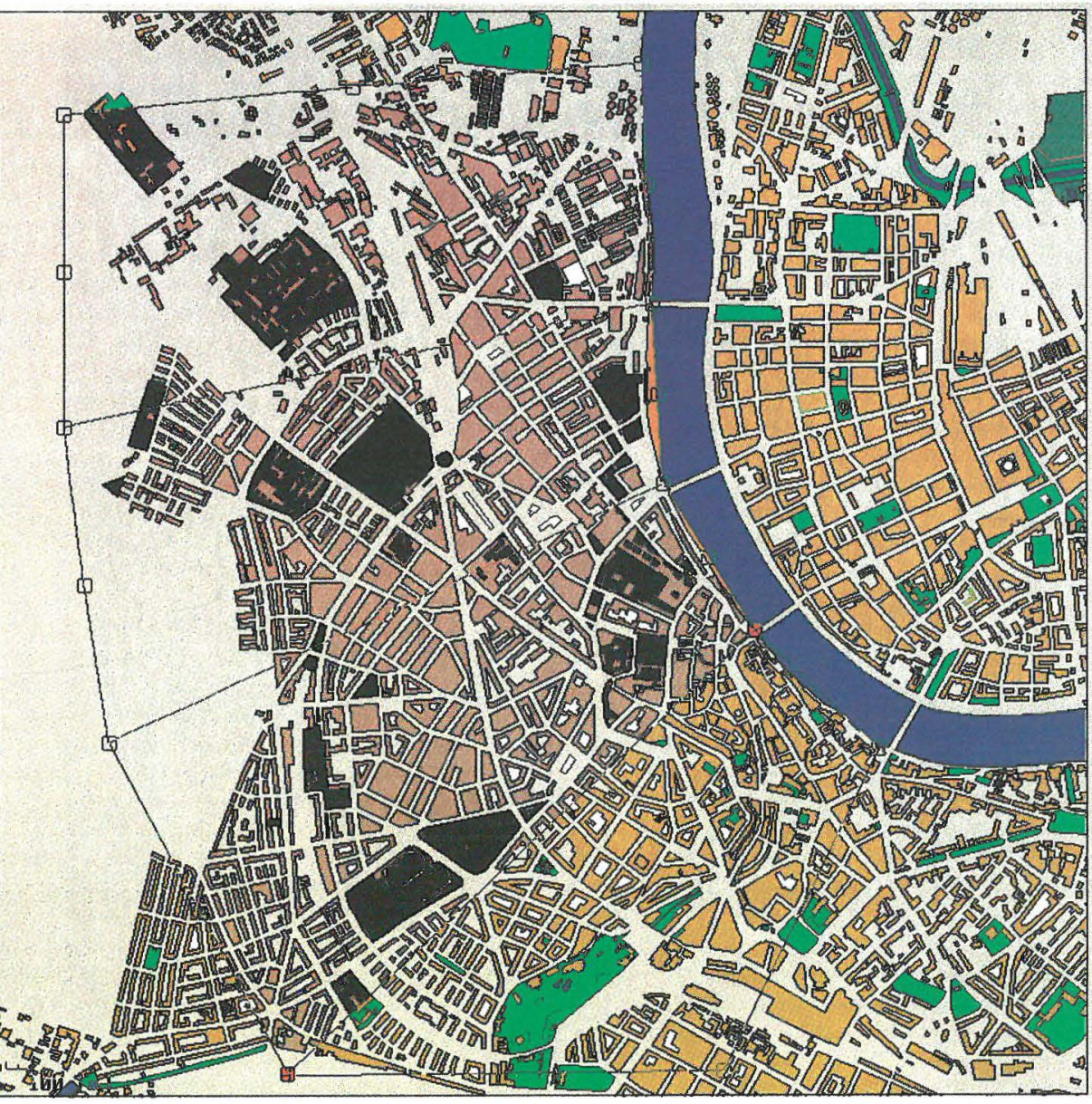

Move the current node with the mouse, set the node by pressing the left button 
a RISICO BEHEERSING Window size

29.3 by $35.1 \mathrm{~km}$

weather station

chlorine production

chlorine use
dump site

dump site

inland waters
marsh land

dunes

scrub

forest

industrial araa

residential ared

buildings

gloss hou

seg walls
highways
rogds

rogds
railways

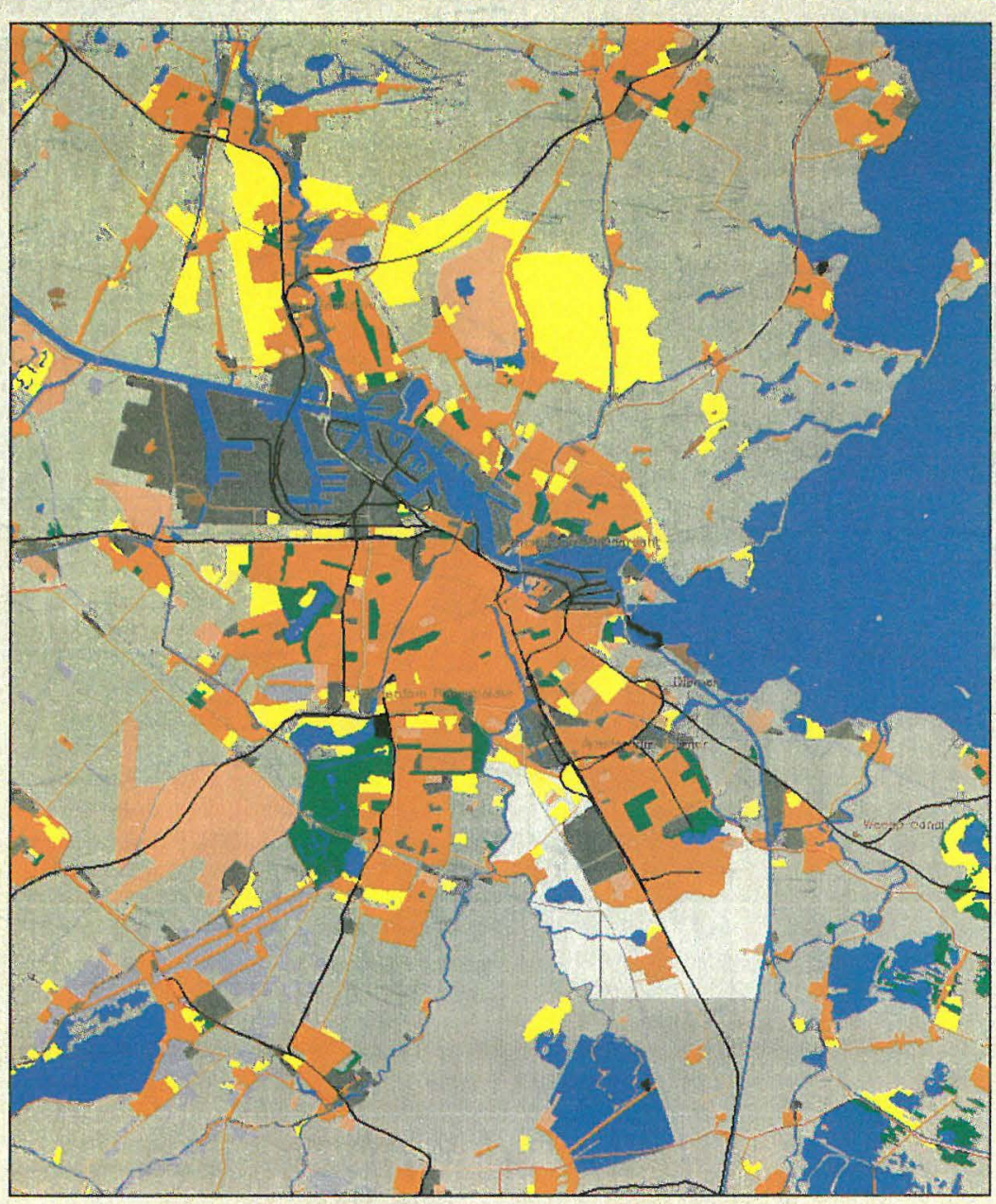

Overlays

VROM specialties

polyline evaluation

toggle population display

togale station-name display

select sub-area (zoom)

undo last transformation

restore original image

weather stations \& wind

chlorine production \& use

EXPLAIN MENU OPTIONS

EXIT REGIONAL DATABASE

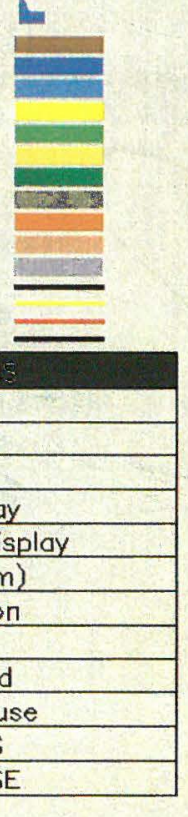


object and a statistical object, which was essential for the risk evaluation. The available data was transformed into three different types of data sources accessible from the system:

(i) spatial information in vector format: data on land use and the road network; the vector format was necessary to allow zooming into the map;

(ii) spatial information in raster format: data on railways, hydrology and administrative boundaries, stored as rasters; and

(iii) relational database: statistical information was related to the areas, arcs and nodes (e.g. population data to the communes (areas), data on products to the firms dealing with the product, the firms to areas or nodes, and number of lanes, road type (arcs)). Further, the area affected by an accident on a specific arc had to be provided within a given corridor, in terms of types of land use affected.

The transportation risk is described by the probability of an accident and the population in the affected area derived from the statistical and geographical data. The risk factor depends on the properties stored for the arc considered (number of lanes, regular maintenance, etc.). A path generator calculates the minimum risk path which is highlighted on the basic map on the screen. At the same time the user is provided with detailed information from the database on each arc used.

\section{REPLACE: interfacing a knowledge-based model with a GIS}

Yet another example of how decision support functions can be linked with GIS is the REPLACE (RElational Plant Location and Acquisition Enquiry) system, a module in the Shanxi Province DSS (Fedra, Li et al. 1987). REPLACE is a knowledge based system based on a matching approach. The idea of approaching site suitability as a matching problem is quite old. Earlier ideas developed in the fifties (e.g. Rawstron 1958), were applied in the feasibility studies of the late sixties and early seventies (e.g. Schilling 1968, Pellenbarg et al. 1974) and in some baseline screening techniques for energy facility planning (Hobbs 1984). 'Relational' matching as applied in REPLACE, however, considers more than a simple matching approach. It is based on the methodological assumption that the definition and measurement of a theoretical concept such as 'site suitability', cannot be based on the empirical characteristics of locations, or the empirical similarities of different locations alone. Instead, site suitability is regarded as the result of a matching of the characteristics and interests of an activity (actor), and the properties of the location (object), as the same empirical properties and combinations of properties of locations, can fulfill very different roles for different activities.

Further, even for the same activities, different combinations of locational properties become important if the objectives and characteristics of that activity change and evolve. What REPLACE has achieved is to develop a model-building technique by which these networks of matching and non-matching actor and object properties and objectives can be represented in such a way that once these representations are available, it is possible to assess the site suitability of various locations for various types of activities (Reitsma 1988). A relational matching model can be regarded as a set-theoretic representation of a concept such as site suitability, i.e. as a set of necessary and sufficient conditions in terms of matching activity and locational properties and characteristics. The matching itself then comes down to a processing of an activity-specific rule base and the data for a set of locations stored in a locational data base, the members of which are tested on the locational requirements inferred from the rule-base. Figure 16.2 shows the conceptual scheme of the core REPLACE system. Running the REPLACE system is simple. After determining the locations which have to be part of the matching, and after determining the exact characteristics of the activity, the dimensionality of 
the problem, and possible generalizations, the matching program processes the associated rule-base, collects the locational requirements, and checks the locations on these requirements.

Figure 16.2: The core matching model

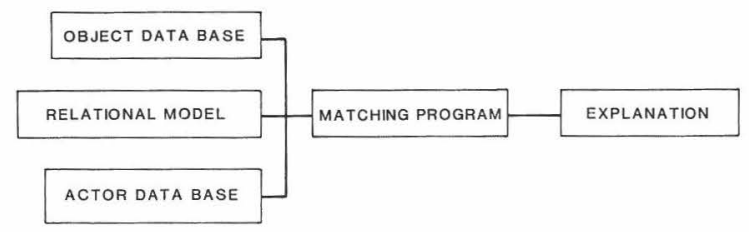

\section{REPLACE and GIS}

REPLACE is a typically spatial model. As such, it is not surprising that both from a modelling and a decision support point of view, interaction with a GIS strongly improves the quality of the analysis. GIS increases REPLACE's usefulness in at least three ways. The first and most obvious way concerns the provision of a set of sophisticated mapping functions. In the current implementation of REPLACE, the mapping is done on an ad hoc basis. Several special-purpose maps are stored on disk or generated dynamically. Although this may be sufficient for a first-draft application, it constitutes a rather inflexible way of dealing with the mapping of matching information and matching results. Using the mapping functions of a GIS, on the other hand, could increase this flexibility significantly, and it would relieve the systems programmer from a great deal of unnecessary work which a GIS can do better anyway. A way in which interaction with a GIS strongly increases the quality of the model results is by using GIS spatial analysis functions for the calculation of locational attributes used in the matching. In many instances, locational data for matching is not available on the level of the areas for which it is matched; either because various standard statistics are collected on different spatial-administrative levels, or because the unit of matching does simply not concur with the areas for which information is available. In such instances, information on one level of spatial organization must be converted to another level, something which may require more-or-less complex overlay techniques. GIS are of course particularly good at this type of operation and can therefore be used to conduct them. Note that this does not require direct interaction between REPLACE and a GIS during matching. Proper calculation of locational attributes can be done prior to matching analysis, and the results can be stored in the locational database system.

For the interpretation of results, standard statistical and, spatial information in the form of maps, tables or graphics, is important as a background. For this type of function, a so-called 
'dedicated GIS' can prove particularly useful. Such a GIS is labelled 'dedicated' because it contains a set of analysis tools tuned to the particular decision support situation at hand (Reitsma and Makare 1989). In the case of REPLACE, for instance, it was realized that a meaningful interpretation of the matching results could be strongly facilitated by the availability of the means to compare the spatial distributions of the matching results with other locational information. For the GIS component of REPLACE therefore, several tasks were recognized and implemented:

(i) uni-variate statistical data description;

(ii) (re)classification of locational attributes;

(iii) bi-variate statistical analysis;

(iv) model results must be treated as a variable; and

(v) mapping on a location-specific basis.

Plate 16.4 shows the screen after the user selects the 'REPLACE data analysis' option from the main REPLACE menu. The screen shows the map of Shanxi Province containing the categorized scores of the attribute displayed on top of the screen, some uni-variate statistics, three frequency histograms, explanation of the colours, and a menu containing further options. Attributes can be selected by choosing the 'Select and display attribute option' from the menu. Three frequency tables are shown: equal interval frequencies, frequencies of the current classification, and cumulative frequencies of the current classification. Attributes are always categorized. Numerical variables are by default categorized in five equal count classes (equal numbers of observations per class). This is reflected in the structure of the two bottom frequency graphs in Plate 16.4. The top frequency graph displays numbers of observations in 50 equal intervals. The bar beneath it corresponds to the current classification. The values associated with the current class boundaries are shown in both the explanation under the map, and the classification frequency graph. In case variables are indeed numerical, uni-variate statistics are displayed. Nominal variables neither need categorization by the system, nor do an equal interval frequency graph and uni-variate statistics apply. These are therefore not displayed. Missing values ('unknown' information) are displayed as white counties on the map.

\section{Re-classification and bi-variate statistics}

In order to enable the user to reclassify numerical attributes into a categorization that is more suited to the purposes, a special graphical reclassification routine was developed. Results become available through automatic updating of the map and the frequency graphs, immediately after reclassifying the attribute. Another feature of the GIS component of REPLACE is the opportunity to calculate some bi-variate associations between variables. At present only a simple, two-variable linear regression and a chi-square test for statistical independence have been implemented, but these two do generate some interesting possibilities for the exploration of statistical relations, especially when combined with maps showing the spatial distributions of their variables. Naturally, regression can only be conducted for interval and ratio variables. If regression is requested for a problem containing a nominal or ordinal variable, the system will tell the user that that is not a desirable option. Whereas regression can be used for numeric attributes, a chi-square test for statistical independence can be applied to a combination of categorized variables. And since, for mapping reasons, numeric variables are also categorized, they can be included in a chi-square test as well. Statistically there is of course no reason to conduct a chi-square test on two numeric variables, but combinations of a numeric variable with a nominal one can be interesting. The dedicated GIS in REPLACE also offers the opportunity to treat matching results as locational variables. This provides the user with a possibility to inspect model results, not only in terms of how they were derived in the first 
place (REPLACE provides the user with an explanation of what happened during the matching process), but also by comparing them with the spatial distributions of other variables, existing patterns of activity location, or other model results.

\subsection{Conclusion}

As the above examples illustrate, there are numerous ways of interlinking GIS with DSS to various degrees whenever a decision-making problem has some spatial dimension. Visualization of a problem's context and structure and its alternative solutions is one of the most powerful components of decision support. Maps are not only a familiar and easy-to-interpret format, but provide an ideal vehicle for the organization of complex spatial information when combined with other symbolic and graphical forms of representations. These symbolic and graphical representations of alternative options can in turn either be retrieved from data bases with or without statistical processing and interpretation, can be generated by appropriate models, or are interactively defined by the user. GIS and DSS have a lot in common, but they also can complement each other in many applications. Merged with simulation and optimization models, and $\mathrm{AI} /$ expert systems technology, they are important building blocks for a new generation of useful and usable 'smart' information technology supporting planners, managers and decision makers.

\section{References}

Barr, A. and Feigenbaum, E.A. (1982) The Handbook of Artificial Intelligence, Volume II, Pitman, London

Bell, D.E., Keeney, R.L. and Raiffa, H. (eds) (1977) Conflicting Objectives in Decisions, International Series on Applied Systems Analysis, John Wiley

Doukidis, G.I., Land, F. and Miller, G. (1989) Knowledge Based Management Support Systems, Ellis Horwood Ltd, Chichester

Fedra, K. (1985) Advanced Decision-oriented Software for the Management of Hazardous Substances. Part I: Structure and Design, CP-85-18; Part II: A Prototype Demonstration System, CP-86-10, International Institute for Applied Systems Analysis, Laxenburg, Austria

Fedra, K. and Diersch, H-J. (1989) Interactive groundwater modeling: color graphics, ICAD and AI, in Proceedings of the International Symposium on Groundwater Management: Quantity and Quality, Benidorm, Spain (2-5 October)

Fedra, K. and Loucks, D.P. (1985) Interactive computer technology for planning and policy modeling, Water Resources Research, 21(2), 114-122

Fedra, K. and Otway, H. (1986) Advanced Decision-oriented Software for the Management of Hazardous Substances. Part III: Decision Support and Expert Systems: Uses and Users, CP-86-14, International Institute for Applied Systems Analysis, Laxenburg, Austria

Fedra, K., Karhu, M., Rys, T., Skoc, M., Zebrowski, M. and Ziembla, W. (1987) Model-based Decision Support for Industry--Environment Interactions. A Pesticide Industry Example, WP-87-97, International Institute for Applied Systems Analysis, Laxenburg, Austria

Fedra, K., Li, Z., Wang, Z. and Zhao, C. (1987) Expert Systems for Integrated Development: A Case Study of Shanxi Province, The People's Republic of China, SR-87-1, International Institute for Applied Systems Analysis, Laxenburg, Austria

Fedra, K., Weigkricht, E., Winkelbauer, L. (1987) A Hybrid Approach to Information and Decision Support Systems: Hazardous Substances and Industrial Risk Management, RR-87-12, International Institute for Applied Systems, Laxenburg, Austria (Reprinted 
from Economics and Artificial Intelligence, Pergamon Books Ltd)

Fick, G. and Sprague, R.H., Jr. (eds) (1980) Decision Support Systems: Issues and Challenges, Proceedings of an International Task Force Meeting (June 23-25), IIASA Proceedings Series, Pergamon Press, Oxford

Hobbs, B.F. (1984) Regional energy facility models for power system planning and policy analysis, in B. Lev et al., Analytic Techniques for Energy Planning, Elsevier Science Publishers, B.V. Amsterdam, pp. 53-66

Keeney, R.L. and Raiffa, H. (1976) Decisions with Multiple Objectives: Preferences and Values Tradeoffs, Wiley, New York

O'Brian, W.R. (1985) Developing "Expert Systems": contributions from decision support systems and judgement analysis techniques, R\&D Management, 15(4), 293-303

Pearl, J., Leal, A. and Saleh, J. (1982) GODDESS: A Goal Directed Decision Supporting Structuring System, IEEE Trans. Pattern Analysis and Machine Intelligence, PAMI, 4(3), 250-262

Pellenbarg, P., Schuurmans, F. and Wouters, J. (1974) De Ontwikkelmogelijkheden van Medemblik: Proeve van een Feasibility Study. 3. The development possibilities of Medemblik: a feasibility study, Geografische Instituut, Rijks Universiteit Groningen, The Netherlands

Phillips, L. (1984) Decision support for managers, in H. Otway and M. Peltu (eds) The Managerial Challenge of New Office Technology, Butterworths, London, p246 Radford, K.J. (1978) Information Systems for Strategic Decisions, Reston Publishing Co. Inc., VA

Rawstron, E.M. (1958) Three principles of industrial location, Transactions and Papers of the IBG, 25, 132-142

Reitsma, R. and Makare, B. (1989) Integration of model-based decision support and dedicated Geographical Information Systems, Journal of Geographical Information Systems (in preparation)

Reitsma, R.F. (1988) REPLACE: The Application of Relational Methodology in Site Suitability Analysis in the IIASA-ACA Shanxi Province Decision Support System, Paper presented at the Annual Conference of the Institute of British Geographers, Loughborough, UK, (January 5-8)

Sage, A.P. and White, C.C. (1984) ARIADNE: a knowledge-based interactive system for planning and decision support, IEEE Transactions on Systems, Man, and Cybernetics, 14(1), 35-47

Schilling, H. (1968) Standortfaktoren fur die Industrieansiedlung; ein Katalog fur die Regionale und Kommunale Entwicklungspolitik sowie die Standortwahl von Unternehmungen (Siting factors for industrial locations and locational choice by enterprises: a handbook for regional and communal policy making), Osterreichisches Institut fur Raumplanung, Veroffentlichungen Nr.27, Kohlhammer GmbH, Stuttgart

Technica (1984) The SAFETI Package. Computer-based System for Risk Analysis of Process Plant, Vol.I-IV and Appendices I-IV, Technica Ltd., Tavistock Sq., London

Wierzbicki, A. (1983) A mathematical basis for satisficing decision making, Mathematical Modeling USA, 3, 391-405 (also appeared as RR-83-7, International Institute for Applied Systems Analysis, A-2361 Laxenburg, Austria)

Zebrowski, M., Dobrowolski, G., Rys, T., Skocz, M. and Ziembla, W. (1988) Industrial structure optimization: the PDAS model, in K. Fedra (ed) Expert Systems for Integrated Development: A Case Study of Shanxi Province, The People's Republic of China. Final Report Volume I: General System Documentation

Zimmermann, H-J. (1987) Fuzzy Sets, Decision Making and Expert Systems, Kluwer Academic Publishers 
Kurt Fedra

Advanced Computer Applications (ACA)

International Institute for Applied Systems Analysis (IIASA)

A-2361 Laxenburg

Austria

Rene F. Reitsma

Katholieke Universiteit Nijmegen

Geografisch Instituut

Thomas van Aquinostraat 5

$6500 \mathrm{KD}$ Nijmegen

The Netherlands 